\title{
Évaluation, reconnaissance des acquis et technologie : tendances en éducation
}

Federica Minichiello

\section{OpenEdition}

\section{Journals}

Édition électronique

URL : https://journals.openedition.org/ries/6254

DOI : $10.4000 /$ ries. 6254

ISSN : 2261-4265

Éditeur

France Education international

Édition imprimée

Date de publication : 1 septembre 2018

Pagination : 11-14

ISBN : 978-2-85420-619-7

ISSN : $1254-4590$

Référence électronique

Federica Minichiello, «Évaluation, reconnaissance des acquis et technologie : tendances en

éducation ", Revue internationale d'éducation de Sèvres [En ligne], 78 I septembre 2018, mis en ligne le 01 septembre 2018, consulté le 25 juin 2021. URL : http://journals.openedition.org/ries/6254 ; DOI : https://doi.org/10.4000/ries.6254

Ce document a été généré automatiquement le 25 juin 2021

(C) Tous droits réservés 


\title{
Évaluation, reconnaissance des acquis et technologie : tendances en éducation
}

\author{
Federica Minichiello
}

1 La dernière décennie aura vu paraître plusieurs évolutions technologiques qui facilitent la reconnaissance des acquis d'expériences non formelles, dans une volonté de continuité avec l'éducation formelle : les MOOC ont ouvert l'accès aux cours, l'échange et l'évaluation entre apprenants à une échelle massive et mondiale, les badges ont introduit une nouvelle liberté dans l'attestation de compétences, les chaînes de blocs (blockchains) annoncent "une désintermédiation et une nouvelle ubiquité de la certification $»^{2}$.

2 S'agit-il d'une utopie ou d'une réalité imminente? Cet article présente certains changements induits par ces technologies dans les domaines de l'évaluation et de la certification et dans les rapports humains sous-jacents : la relation avec soi-même, l'échange de pair à pair, la position vis-à-vis d'un évaluateur ou d'un établissement certificateur, encore détenteurs d'une légitimité qui prédomine sur une approche exclusivement technologique.

3 Malgré une valeur ajoutée assez intuitive et des expériences d'application encourageantes, il demeure difficile de prévoir l'importance qu'étudiants, employeurs ou institutions éducatives attribueront à ces technologies et à l'élément au cœur de ce système, la souveraineté sur les données.

4 Sitographie arrêtée le 20 juillet 2018. 


\section{Traces numériques et MOOC}

\section{« Auto-évaluation : l'aide du numérique aux activités d'autoévaluation » Mailles-Viard Metz}

5 Cet article de 2015 paru dans la Revue internationale de pédagogie de l'enseignement supérieur illustre l'impact des technologies sur l'analyse réflexive d'un apprenant sur ses activités et sur les pratiques d'auto-évaluation. Dans un univers parsemé de traces numériques, enregistrées par des systèmes d'information ou laissées volontairement par un utilisateur, la lecture ou l'interprétation de ces traces reste un exercice relativement compliqué et peu accessible; l'apprentissage adaptif (learning analytics) est pour l'instant pensé pour une interprétation par un enseignant, sans que l'apprenant ait prise sur ces éléments. Des travaux de recherche explorent une meilleure autonomisation de l'apprenant, afin que les environnements d'apprentissage lui restituent davantage de maîtrise, dans un esprit de " réflexivité augmentée ». https://journals.openedition.org/ripes/991

\section{"Une analyse automatisée des modalités d'évaluation dans les MOOC »}

6 Cet article de M. Cisel $^{3}$ part du constat que la certification d'un MOOC est souvent utilisée dans un curriculum, malgré des méthodologies d'évaluation très variables. À partir de l'analyse de 131 cours organisés en 2014-2015 sur la plateforme française FUN, l'auteur montre que la majorité des concepteurs de MOOC ne recourt pas à de l'évaluation par les pairs, une pratique auparavant réservée à la publication scientifique et que les MOOC ont démocratisée. Les cours intégrant l'évaluation par les pairs afficheraient par ailleurs des taux de certification plus bas que la moyenne. Les concepteurs préfèreraient recourir à des modalités d'évaluation peu exigeantes, conscients que la certification d'un MOOC revêt d'autres fonctions que la validation formelle d'acquis: l'autoévaluation, une meilleure appropriation de principaux messages des cours, etc.

http://www.ijede.ca

\section{OpenCred}

7 OpenCred est une étude menée en 2014 par l'Université de Leicester dans le cadre du projet OpenEdu, en collaboration avec le Centre commun de recherche (Joint Research Center: JRC), sur les pratiques d'évaluation de l'apprentissage non formel dans des MOOC. Les principales barrières en termes de reconnaissance identifiées en 2014 restent d'actualité : une faible appréciation de la valeur de l'évaluation en ligne et l'absence d'intégration avec les instruments déjà en place de mobilité et de reconnaissance des acquis. L'étude préconise que les plateformes de MOOC délivrent essentiellement des badges ${ }^{4}$, pour encourager le taux de participation et une ludification de l'apprentissage, dans l'idée que les badges pourraient revêtir, au fil des années, une place prépondérante dans le processus de récompense du travail effectué. https://ec.europa.eu/jrc/ 


\section{Badges et Blockchain}

\section{Open Badge}

8 Un badge est un indicateur d'un travail accompli, d'une compétence, d'une qualité. D'un point de vue strictement technique, c'est une image numérique dans laquelle sont enregistrées un certain nombre de métadonnées, notamment les identités de l'émetteur et du récepteur, les critères et les preuves d'attribution. Cette technologie très simple contient cependant une richesse d'informations impossibles à altérer, grâce à des techniques de cryptographie. L'initiative "Open badges", lancée par les fondations Mozilla et MacArthur en 2011, a permis de définir un standard technique " ouvert " pour permettre l'interopérabilité des badges, afin que toute organisation puisse en émettre et en vérifier l'authenticité. Cette initiative a été reprise en 2017 par le consortium international IMS, avec le lancement d'une nouvelle spécification « Open badge 2.0 ", qui permet désormais à des badges ouverts d'être endossés par un tiers (personne ou institution) : ainsi, un badge délivré par une structure locale peut être reconnu et endossé par un organisme national ou international qui délivre un diplôme. En Europe, un partenariat stratégique a été financé entre 2014 et 2017, dans le cadre du programme Erasmus+, pour promouvoir un écosystème qui favorise l'utilisation de badges ; parmi les structures impliquées, on peut citer l'université Beuth, à Berlin, qui a notamment travaillé sur l'utilisation des badges pour la reconnaissance de compétences de personnes en situation de migration.

https://www.imsglobal.org/;

https://openbadges.org/;

http://www.openbadgenetwork.com/ ;

https://beuthbadges.wordpress.com/

\section{Association Reconnaître}

Cette association ${ }^{5}$ a pour objectif de "construire une société ouverte et apprenante fondée sur la reconnaissance des talents, des compétences et des aspirations ». Elle porte actuellement le projet MIRVA, parrainé par la Commission européenne, pour « rendre la reconnaissance informelle visible et réalisable » dans la perspective, d'ici 2020, d'établir un cadre de la reconnaissance ouverte et des lignes directrices pour l'ensemble des parties prenantes. Le président de cette association, S. Ravet, a écrit de nombreux articles sur les potentialités des badges pour construire « une société de la reconnaissance ». Un badge peut être lié à d'autres dans une " chaîne de badges ", une sorte de base de données d'acquis distribuée sur Internet, où « tout peut faire l'objet d'un badge, tout est relation ». Dans son article "Réflexions sur la genèse des Open Badges » $(2018)^{6}$, l'auteur constate deux faiblesses initiales du système : l'absence d'une réflexion sur l'expérience de l'utilisateur (« comme si, pour gérer un courrier, on devait avoir une application pour stocker et lire, l'autre pour l'écrire ») et une position idéologique réservant la légitimité d'émettre des badges à des autorités reconnues, une vision désormais dépassée par l'Open Badge 2.0 (cité supra). L'image ludique souvent associée aux badges minimiserait leur valeur en tant que véritable outil de reconnaissance. Mais ils sont pourtant utilisés par des établissements d'enseignement supérieur, comme, le consortium interuniversitaire italien CINECA. 
https://reconnaitre.openrecognition.org ;

https://bestr.it/

\section{"Comprendre les blockchains »}

10 La note "Comprendre les blockchains" (2018) de l'office parlementaire français d'évaluation des choix scientifiques et technologiques ${ }^{7}$ définit la "chaîne de blocs " comme une technologie de stockage et de transmission d'informations, qui permet « la constitution de registres répliqués et distribués " sans le recours à un organisme régulateur central, «structurés par des blocs liés les uns aux autres et sécurisés grâce à la cryptographie». Dans une blockchain on ne peut pas altérer le contenu d'une transaction, chaque transaction étant stockée dans un bloc horodaté et validé en moyenne toutes les dix minutes. Si cette technologie est surtout connue pour les cryptomonnaies (Bitcoin, Ether, etc.) les possibilités d'application fleurissent mais, selon la note, "peu conjuguent, à ce jour, maturité technologique suffisante et pertinence d'usage ».

http://www.senat.fr/opecst

\section{EU Blockchain Observatory and Forum}

11 La Direction générale Réseaux de communication, contenu et technologies (DG CONNECT) considère l'intelligence artificielle et la blockchain comme les deux technologies révolutionnaires du moment. La Commission européenne a créé en février 2018 un Observatoire-forum dédié pour analyser et cartographier l'écosystème en Europe et capitaliser sur les initiatives existantes, parallèlement à un appui financier à hauteur de 340 millions d'euros dans le cadre du programme de recherche Horizon 2020. Selon l'Observatoire, l'éducation ferait partie des domaines d'activité les plus concernés par cette technologie, pour ce qui relève des diplômes et des certifications : l'empreinte numérique cryptée d'un diplôme serait stockée dans une transaction, au cœur d'une blockchain, permettant la vérification à toute personne autorisée, à l'abri de tentatives de piratage.

https://www.eublockchainforum.eu

\section{«Blockchain in Education »}

Ce rapport 2017 du Centre commun de recherche détaille l'application de la blockchain pour stocker des qualifications et des attestations de réussite, voire automatiser la reconnaissance des diplômes et le transfert des crédits, sans la nécessité de recourir à l'organisme émissaire ou à des tiers intermédiaires. Chaque individu garderait une maîtrise sur ses propres données en ouvrant l'accès à qui de droit selon les besoins. Il s'agit d'un défi de taille car, comme le rapport l'indique, les outils existants pour favoriser la mobilité (supplément au diplôme, ECTS, etc.) n'ont pas été pensés pour être lus par des machines.

Les principales difficultés à surmonter seraient la définition du modèle économique (plus facile à construire autour de la propriété) et la complexité d'utilisation, qui pourrait nécessiter la conservation d'intermédiaires et, par conséquent, un maintien de la logique de propriété, notamment intellectuelle. Plusieurs études de cas d'utilisation sont présentées : le Massachusetts Institute of Technology, l'Université de Nicosie, des 
établissements d'enseignement à Malte.

http://publications.jrc.ec.europa.eu/

\section{Edublock}

Learning is Earning 2026 est un jeu prospectif organisé par l'Institute for the Future et ACT Foundation pour ré-imaginer, pendant 36 heures, le futur du travail et de l'apprentissage. «Un bloc, une heure d'apprentissage »-formel ou informel : telle est la devise de l'edublock, un système de monnaie virtuelle où chaque bloc gagné et crédité permettrait à son détenteur d'enseigner à son tour les compétences à peine acquises. À partir de l'idée que la réputation a déjà une valeur économique, l'Open university a, de son côté, imaginé un système dans lequel chaque institution reçoit une quantité définie de devise intellectuelle («Kudos») et la redistribue à son personnel, chacun stockant ses crédits dans la blockchain. Chaque individu peut publier son travail, des tiers peuvent lui attribuer une valeur matérialisée en crédit Kudos, comme dans un système de financement participatif où la réputation devient la monnaie. Les pratiques d'évaluation par les pairs et de réputation, auparavant réservées au monde académique, se généraliseraient au plus grand nombre, avec des zones d'ombres qui restent à éclaircir : l'éventuel taux de conversion en argent, le risque d'une ouverture de la connaissance qui pourrait vite se transformer en marchandisation de la connaissance.

http://www.learningisearning2026.org/

\section{NOTES}

2. JRC Science for Policy report : «Blockchain in Education » (2017) (ce rapport est présenté plus loin).

3. M. Cisel. "Une analyse automatisée des modalités d'évaluation dans les MOOC » in Revue internationale d'apprentissage en ligne et de l'enseignement à distance [en ligne] vol $33 \mathrm{n}^{\circ} 1$ (2018). Voir également les blogs: http://blog.educpros.fr/matthieu-cisel et http:// www.matthieucisel.fr/.

4. Pour une définition des badges, voir infra.

5. Association qui compte, parmi ses membres fondateurs, l'Espace Mendès France, le Centre de recherches interdisciplinaires (CRI), la Ligue de l'enseignement, etc.

6. S. Ravet [en ligne] : https://www.innovation-pedagogique.fr/article2885.html (2018).

7. Délégation indépendante, au sein du Parlement français, commune à l'Assemblée nationale et au Sénat. 
INDEX

Mots-clés : reconnaissance des acquis, certification, badge, chaîne de blocs, blockchain Palabras claves : validación de conocimientos anteriores, certificación, badge, cadena de bloques, blockchain

Keywords : prior learning recognition, certification, badges, blockchain

\section{AUTEUR}

\section{FEDERICA MINICHIELLO}

Federica Minichiello est responsable adjointe du Centre de ressources et d'ingénierie documentaires du Centre international d'études pédagogiques. Courriel : minichiello@ciep.fr 